Broman-Fulks, J. J., \& Storey, K. M. (2008). Evaluation of a brief exercise intervention for high anxiety sensitivity. Anxiety, Stress, and Coping, 21(2): 117-128. (Jun 2008) Published by Taylor \& Francis (ISSN: 1477-2205). DOI: 10.1080/10615800701762675

\title{
Evaluation of a brief aerobic exercise intervention for high anxiety sensitivity
}

\author{
Joshua J. Broman-Fulks and Katelyn M. Storey
}

\begin{abstract}
Anxiety sensitivity, or the belief that anxiety-related sensations can have negative consequences, has been shown to play an important role in the etiology and maintenance of panic disorder and other anxiety-related pathology. Aerobic exercise involves exposure to physiological cues similar to those experienced during anxiety reactions. The present study sought to investigate the efficacy of a brief aerobic exercise intervention for high anxiety sensitivity. Accordingly, 24 participants with high anxiety sensitivity scores (Anxiety Sensitivity Index-Revised scores $>28$ ) were randomly assigned to complete either six 20-minute sessions of aerobic exercise or a no-exercise control condition. The results indicated that individuals assigned to the aerobic exercise condition reported significantly less anxiety sensitivity subsequent to exercise, whereas anxiety sensitivity scores among non-exercisers did not significantly change. The clinical research and public health implications of these findings are discussed, and several potential directions for additional research are recommended.
\end{abstract}


Anxiety sensitivity, or the fear of anxiety and anxiety-related sensations, has been shown to play an important role in the development and maintenance of panic attacks, panic disorder, and other anxiety and Axis I disorders (e.g., Ehlers, 1995; Schmidt, Lerew, \& Jackson, 1997; Schmidt, Zvolensky, \& Maner, 2006). As such, researchers have begun to search for interventions that can effectively reduce anxiety sensitivity, and, in turn, minimize the incidence of various forms of psychopathology. To date, the vast majority of the anxiety sensitivity treatment literature has focused on investigating the effects of cognitive-behavioral therapy (CBT) on anxiety sensitivity, and the results have been promising. For example, several studies have found that 10 to 12 sessions of CBT can generate significant reductions in anxiety sensitivity among patients with anxiety disorders (e.g., McNally \& Lorenz, 1987; Telch et al., 1993). In addition, 2 days of intensive CBT (i.e., 9 hours) have also been shown to produce a significant drop in anxiety sensitivity scores among panic patients (Deacon \& Abramowitz, 2006). Most recently, a brief CBT paradigm, consisting primarily of psychoeducational components, was shown to significantly reduce anxiety sensitivity and risk for the development of Axis I disorders at 2-year follow-up (Schmidt et al., 2007).

Aerobic exercise has been shown to be an effective and cost-efficient treatment alternative for a variety of anxiety and mood disorders, including panic disorder (for a review, see Salmon, 2001). Noting that most CBT protocols for anxiety sensitivity and panic generally involve interoceptive exposure and arousal reduction components, Broman-Fulks, Berman, Rabian, and Webster (2004) recently suggested that aerobic exercise may be an alternative mode of affecting anxiety sensitivity through similar processes. Specifically, an integral aspect of many CBT treatments for panic and anxiety involves interoceptive exposure, or the intentional, repeated induction of anxious arousal symptoms via behavioral techniques. Aerobic exercise generates many of the same physiological sensations that are elicited during anxiety reactions, including elevations in heart rate, respiration, and perspiration. Thus, repeated exposure to anxiety-related interoceptive cues via physical exercise may serve to extinguish fears of these sensations in similar ways as other interoceptive exposure techniques (Broman-Fulks et al., 2004). In addition, similar to the arousal reduction components of CBT (e.g., breathing retraining, relaxation exercises), aerobic exercise has been shown to reduce general arousal levels, including resting heart rate and muscle tension (Abadie, 1988). Indeed, several studies have indicated that aerobic exercise may be as effective as CBT in reducing generalized anxiety (e.g., Fremont \& Craighead, 1987; McEntee \& Haglin, 1999).

Despite the apparent similarities between aspects of CBT for panic and aerobic exercise, only one study, to date, has attempted to empirically evaluate the effects of aerobic exercise on anxiety sensitivity. In an exploratory study, Broman-Fulks and colleagues (2004) randomly assigned 54 participants to complete six 20-minute sessions of high-intensity aerobic exercise or low-intensity, non-aerobic activity. The results indicated significant declines in anxiety sensitivity for both treatment conditions at follow-up. However, the aerobic exercise protocol produced more rapid reductions in anxiety sensitivity and more treatment responders (defined as a decrease in ASI scores of $>1$ SD) than the non-aerobic activity. Furthermore, only high-intensity aerobic exercise produced significant reductions in fears of anxiety-related physical sensations. Thus, initial evidence appears to indicate that aerobic exercise may serve as an effective, cost-efficient alternative to CBT for reducing anxiety sensitivity.

The findings reported by Broman-Fulks et al. (2004) represent an important first step in evaluating aerobic exercise as a potential intervention for anxiety sensitivity. However, due 
to the preliminary nature of these findings, additional research is needed and replication is necessary. In addition, several important methodological limitations need to be addressed. For example, although inclusion of an active control condition was commendable, both exercise conditions in the Broman-Fulks et al. (2004) study reported significant declines in anxiety sensitivity scores, thus preventing the elimination of statistical regression as an explanation for their findings. In addition, although fears of anxiety-related physical symptoms were evaluated at each exercise session, the previous report only assessed the full domain of anxiety sensitivity at baseline, after 2 weeks of exercise, and at an additional 1-week follow-up, thus preventing detailed observation of change in anxiety sensitivity scores over time. Furthermore, the effects of aerobic exercise on the various subcomponents of anxiety sensitivity were not directly examined.

The purpose of the present study was to address some of these issues and further evaluate the efficacy of aerobic exercise as an intervention option for individuals with high anxiety sensitivity. To this end, individuals with high anxiety sensitivity were randomly assigned to complete six 20-minute sessions of high-intensity aerobic exercise or a no-exercise control condition. A comprehensive measure of anxiety sensitivity, which also enabled a detailed analysis of the subcomponents of anxiety sensitivity, was administered to participants at baseline, following each session, and at a 1-week follow-up appointment. Based on previous research, it was hypothesized that individuals assigned to the aerobic exercise condition would report significantly greater improvements in overall and subscale anxiety sensitivity scores in comparison with those assigned to the no-exercise control condition.

\section{METHOD}

\section{Participants}

Participants were recruited from undergraduate psychology courses at Appalachian State University. Thirty-five students (28 females), ages 18 through 27 (M_18.91, SD_1.62), met inclusion criteria and agreed to participate in this study in exchange for course credit. To be included in the study, participants had to: (a) achieve a score above the non-clinical mean (i.e., >26; Deacon, Abramowitz, Woods, \& Tolin, 2003) on the Anxiety Sensitivity Index-Revised (ASI-R; Taylor \& Cox, 1998); (b) be at least 18 years of age; and (c) be in good physical health (assessed using the Physical Activity Readiness Questionnaire, PAR-Q; Shephard, Cox, \& Simper, 1981). Exclusion criteria were: (a) any health condition that would preclude aerobic exercise; (b) current involvement in psychotherapy; (c) current use of psychiatric medications; or (d) current involvement in an aerobic exercise program. Current exercise involvement was defined as more than one exercise session per week. Of the 35 participants who met criteria and agreed to participate, 24 (19 females) completed the entire study through follow-up ( $M$ age=19.04, $S D=1.90$ ). Thus, the remaining analyses are for those 24 completers unless noted otherwise.

\section{Instruments}

The ASI-R (Taylor \& Cox, 1998) is a revised and expanded version of the original 16-item Anxiety Sensitivity Index, consisting of 36 items that are rated on a five-point Likert Scale, ranging from (0) "very little" to (4) "very much." Factor analysis has identified four lower order factors, which have been labeled: Fear of Respiratory Symptoms, Fear of Publicly 
Observable Anxiety Reactions, Fear of Cardiovascular Symptoms, and Fear of Cognitive Dyscontrol (Taylor \& Cox, 1998). Construct validity for the ASI-R has been established based on significant correlations with the original ASI ( $r=.94$; Taylor \& Cox, 1998). The ASI-R has also been shown to display adequate criterion validity, with anxiety disorder patients tending to score higher than individuals with no history of anxiety disorders (e.g., Beck \& Wolf, 2001). The ASI-R has demonstrated excellent internal consistency (alpha $=.95)$, with all 36 items showing adequate item-total correlations $(M=.58$, range $=.40-.71$;

Deacon et al., 2003).

\section{Procedure}

The present study was reviewed and approved by the Institutional Review Board at Appalachian State University. Prospective participants completed a screening consent form, the ASI-R, and a brief demographic questionnaire. Individuals who met the initial selection criteria were administered the PAR-Q to ensure their safety and determine if they were healthy enough for beginning an exercise regimen. Individuals who reported health problems that would preclude exercise were excused from the study. Individuals who met the inclusion/exclusion criteria were asked to complete the informed consent process. Those who agreed to participate were randomly assigned to either a high-intensity aerobic exercise or no-exercise control condition. All participants were told that they would be completing a study designed to examine the relationship between college student exercise habits and various mood states over time. Participants in both conditions were unaware of the other condition, and only individuals assigned to the experimental condition were aware that the experiment involved direct physical exercise.

Participants in the exercise group completed an aerobic exercise regimen designed to expose them to the bodily sensations presumably associated with anxiety. The exercise protocol was designed to replicate methodology used in previous research, indicating that six sessions of aerobic exercise can significantly reduce anxiety sensitivity scores (BromanFulks et al., 2004). Thus, exercise participants completed six 20-minute aerobic exercise sessions over a 2-week period, with no fewer than two and no more than four sessions per week. At each session, exercisers were fitted with a Polar heart rate monitor. Consistent with the recommendations of the American College of Sports Medicine (2000) for aerobic activity, exercise participants were asked to briskly walk or jog on a treadmill at a speed that maintained their heart rate between 60 and $90 \%$ of their predicted maximum heart rate for the full 20-minute session (p. 442). The lower and upper bound for each participant's aerobic heart rate range was computed using the following formula: $(220$-age $) \times(0.60$ [lower bound] or 0.90 [upper bound]). Heart rates were monitored by the experimenter at 2-minute intervals, and treadmill speeds were adjusted as necessary to maintain aerobic heart rates. Participants were not allowed to engage in any other activities while exercising (e.g., talking, singing, listening to music) to minimize distractions from arousal sensations. In addition, the experimenter monitored participants from a separate room, and only entered the exercise room to inspect the participants' heart rate monitor and adjust treadmill speed if necessary. Following each 20-minute exercise session, participants completed a 5-minute cool down period followed by the ASI-R. In contrast, individuals assigned to the no-exercise control condition reported to the lab six times over 2 weeks (no fewer than two and no more than four times per week) just to complete the ASI-R. In addition, all participants, regardless of condition, were asked to report to the laboratory for a 1-week follow-up appointment at which they completed the ASI-R a final time. Thus, 
ASI-R measurements were taken at eight time points (i.e., baseline, six sessions, and 1-week follow-up).

\section{Data Analysis}

The effects of aerobic exercise on ASI-R full-scale and subscale scores were tested using separate group by assessment session $(2 \times 8)$ ANOVAs. Significant interactions were analyzed by examining within-group simple effects and post hoc mean comparisons. All main effects, simple effects, and interaction effects were interpreted using a Type I error rate of .05. Effect sizes were reported using partial eta-square $\left(n 2_{P}\right)$; which represents effect size as a function of the total variance accounted for by the independent variable.

\section{Preliminary Analyses}

Independent t-tests and chi-square analyses indicated that the exercise and control groups were comparable at baseline on all demographic variables and baseline ASI-R scores (all ps >.10; see Table I). Furthermore, consistent with selection for high anxiety sensitivity, baseline ASI-R scores $(M=42.3, S D=10.2)$ were found to be substantially higher than the non-clinical norms noted in previous reports (e.g., $M=25.7, S D=19.6$; Deacon et al., 2003). Analyses comparing completers with non-completers also revealed that the two groups did not differ on any of the demographic characteristics, panic history, baseline ASI-R scores, or baseline to post Session 1 change in ASI-R scores. Although the exercise group experienced nearly twice the number of non-completers ( 7 versus 4 in the control group), this difference did not reach statistical significance $(p>.10)$.

Table I. Demographic characteristics of the aerobic exercise and no-exercise groups.

\begin{tabular}{|c|c|c|c|c|}
\hline \multirow[b]{2}{*}{ Variable } & \multicolumn{2}{|c|}{ Aerobic exercise $(n=12)$} & \multicolumn{2}{|c|}{ No-exercise $(n=12)$} \\
\hline & $n$ & $M(S D)$ & $n$ & $M(S D)$ \\
\hline Age & & $19.33(2.50)$ & & $18.75(1.06)$ \\
\hline \multicolumn{5}{|l|}{ Gender } \\
\hline Men & 3 & & 2 & \\
\hline Women & 9 & & 10 & \\
\hline \multicolumn{5}{|l|}{ Race } \\
\hline Caucasian & 11 & & 11 & \\
\hline African-American & - & & 1 & \\
\hline Asian & 1 & & - & \\
\hline \multicolumn{5}{|l|}{ Panic history } \\
\hline Yes & 2 & & 4 & \\
\hline No & 10 & & 8 & \\
\hline \multicolumn{5}{|l|}{ Completers } \\
\hline Yes & 12 & & 12 & \\
\hline No & 7 & & 4 & \\
\hline
\end{tabular}

Note. The groups did not significantly differ on any of the above variables. 


\section{RESULTS}

\section{Anxiety Sensitivity Analyses}

A $2 \times 8$ (group_session) repeated measures ANOVA was computed on ASI-R total scores to determine whether scores varied over time as a function of group. The results revealed a significant main effect for session, $\mathrm{F}(7,154)=4.92$, pB.001, $\mathrm{n} 2_{\mathrm{p}}=.18$; with ASI-R total scores decreasing from baseline to Session 1 , and then remaining relatively stable through post and follow-up. Table II contains the means and standard deviations of ASI-R full-scale and subscale scores for the two groups at each assessment session. Post hoc analyses indicated that the decrease in ASI-R scores from baseline to each of the subsequent assessment sessions was significant, but that scores did not significantly change following Session 1. Although no significant main effect for group was noted, the results did indicate a significant group by session interaction, $F(7,154)=4.41, p_{-} .001, n 2_{p}=.17$ : A significant simple effect for session emerged for the exercise group, $\bar{F}(7,77)=10.48, p<.001, n 2_{p}=.49$. Post hoc analyses indicated that the exercise group reported a significant decline in ASI-R scores from baseline to the end of Session 1, with lower anxiety sensitivity scores being maintained through follow-up. However, ASI-R scores for the exercise participants did not significantly change following the initial decline in scores post Session 1. In contrast, the control group did not demonstrate an effect for session, $F(7,77)=1.60, p>.10, n 2_{p}=.13$, indicating that control group scores remained relatively stable from baseline through follow-up. Although visual inspection of control group ASI-R scores suggested a modest decline in scores following Session 1 , this change did not reach statistical significance ( $p$ > .10), and scores appeared to return to baseline levels by the end of Session 3. Figure 1 shows a graphical display of the mean change in ASI-R total scores for aerobic exercise and no-exercise groups from baseline to each subsequent session.

Table II. Means and standard deviations of ASI-R total scores and subscale scores for the aerobic exercise and no-exercise groups at baseline, following each exercise session, and at 1-week follow-up.

\begin{tabular}{|c|c|c|c|c|c|c|c|c|c|c|}
\hline & \multicolumn{2}{|c|}{ ASI-R total score } & \multicolumn{2}{|c|}{$\begin{array}{l}\text { Fear of Respiratory } \\
\text { Symptoms }\end{array}$} & \multicolumn{2}{|c|}{$\begin{array}{c}\text { Fear of Publicly Observable } \\
\text { Anxiety Reactions }\end{array}$} & \multicolumn{2}{|c|}{ Fear of Cardiovascular Symptoms } & \multicolumn{2}{|c|}{$\begin{array}{l}\text { Fear of Cognitive } \\
\text { Dyscontrol }\end{array}$} \\
\hline & $\begin{array}{l}\text { Aerobic } \\
\text { exercise }\end{array}$ & No-exercise & $\begin{array}{l}\text { Aerobic } \\
\text { exercise }\end{array}$ & No-exercise & $\begin{array}{l}\text { Aerobic } \\
\text { exercise }\end{array}$ & No-exercise & $\begin{array}{l}\text { Aerobic } \\
\text { exercise }\end{array}$ & No-exercise & $\begin{array}{l}\text { Aerobic } \\
\text { exercise }\end{array}$ & No-exercise \\
\hline Session & $M(S D)$ & $M(S D)$ & $M(S D)$ & $M(S D)$ & $M(S D)$ & $M(S D)$ & $M(S D)$ & $M(S D)$ & $M(S D)$ & $M(S D)$ \\
\hline Baseline & $45.17(7.77)$ & $39.50(11.84)$ & $19.42(6.06)$ & $18.83(8.31)$ & $14.42(3.96)$ & $12.42(4.27)$ & $10.42(5.93)$ & $8.00(4.92)$ & $2.92(3.09)$ & $2.50(2.54)$ \\
\hline Session 1 & $30.75(10.94)$ & $32.83(16.88)$ & $12.58(6.32)$ & $12.75(7.68)$ & $11.42(5.79)$ & $10.33(4.87)$ & $6.33(4.05)$ & $7.58(5.12)$ & $0.42(0.67)$ & $2.33(2.64)$ \\
\hline Session 2 & $29.58(11.84)$ & $33.83(19.47)$ & $12.33(5.73)$ & $11.75(8.30)$ & $11.58(5.60)$ & $12.17(6.07)$ & $5.42(3.80)$ & $7.42(5.63)$ & $0.25(0.62)$ & $2.50(3.90)$ \\
\hline Session 3 & $27.50(13.06)$ & $37.08(21.37)$ & $11.50(6.83)$ & $12.92(8.14)$ & $10.58(5.25)$ & $11.83(6.59)$ & $5.08(3.91)$ & $9.00(7.07)$ & $0.33(0.78)$ & $3.33(3.82)$ \\
\hline Session 4 & $29.50(15.92)$ & $36.42(20.35)$ & $11.92(7.56)$ & $12.58(8.34)$ & $10.75(5.61)$ & $12.08(6.69)$ & $6.42(5.26)$ & $7.83(5.64)$ & $0.42(1.16)$ & $3.92(4.66)$ \\
\hline Session 5 & $28.33(13.80)$ & $37.42(23.38)$ & $11.75(7.55)$ & $13.00(10.21)$ & $10.08(5.20)$ & $12.25(6.89)$ & $6.17(4.75)$ & $8.17(6.63)$ & $0.33(0.78)$ & $4.00(4.75)$ \\
\hline Post & $27.92(15.36)$ & $41.00(25.68)$ & $11.58(7.63)$ & $13.08(9.96)$ & $10.42(5.21)$ & $14.33(9.43)$ & $5.75(4.81)$ & $9.58(8.60)$ & $0.17(0.17)$ & $4.00(4.95)$ \\
\hline Follow-up & $26.83(16.96)$ & $41.92(27.10)$ & $11.17(7.69)$ & $14.42(10.37)$ & $9.50(5.55)$ & $12.67(6.97)$ & $6.00(5.61)$ & $10.50(9.03)$ & $0.17(0.17)$ & $4.33(5.60)$ \\
\hline
\end{tabular}

Note. Aerobic exercise with $n=12$, no-exercise with $n=12$; ASI-R = Anxiety Sensitivity Index-Revised; Fear of Respiratory Symptoms, Fear of Publicly Observable Anxiety Reactions, Fear of Cardiovascular Symptoms, and Fear of Cognitive Dyscontrol =ASI-R subscale scores. 


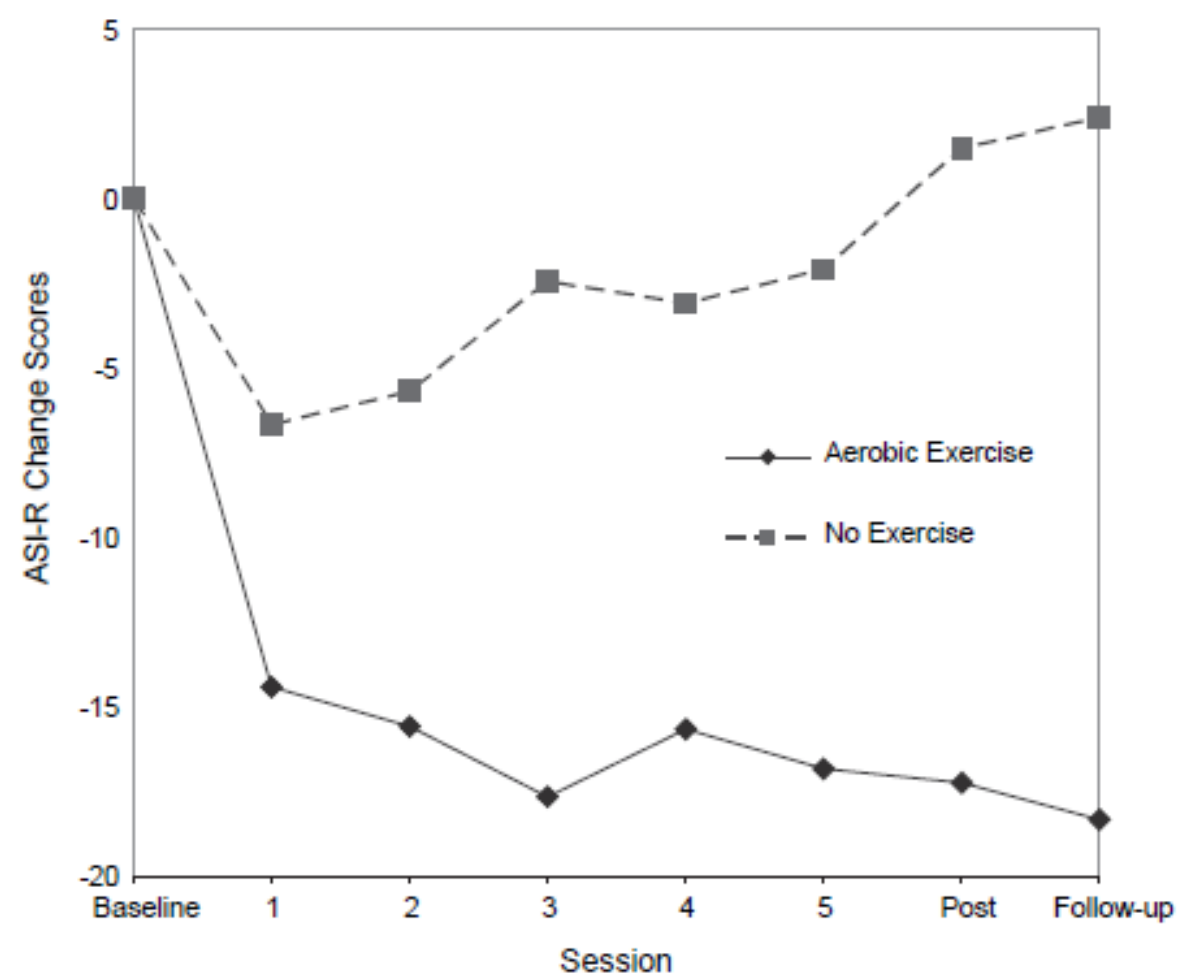

Figure 1. Mean changes in ASI-R total scores for aerobic exercise $(n=12)$ and no-exercise $(n=12)$ groups at baseline, following each exercise session, and at 1-week follow-up. The decline in scores for the aerobic exercise group from baseline to all subsequent sessions was statistically significant at $p<.05$. None of the changes in ASI-R scores for the no-exercise control group reached statistical significance.

\section{ASI-R Subscale Analyses}

As noted above, ASI-R scores can also be broken down into four subscales: Fear of Respiratory Symptoms, Fear of Publicly Observable Anxiety Reactions, Fear of Cardiovascular Symptoms, and Fear of Cognitive Dyscontrol (Taylor \& Cox, 1998). Thus, to examine the relative effects of exercise on each of the dimensions of anxiety sensitivity assessed by the ASI-R, exploratory analyses, consisting of $2 \times 8$ (group_session) repeated measures ANOVAs, were also conducted on ASI-R subscale scores.

Fear of Respiratory Symptoms. The results of a $2 \times 8$ repeated measures ANOVA of the Fear of Respiratory Symptoms subscale revealed a significant main effect for session, $F(7$, 154) $=8.01, \mathrm{pB} .001, \mathrm{n} 2 \mathrm{p}=.27$; with subscale scores decreasing from baseline to Session 1 and remaining relatively stable through post and follow-up. No main effect for group or interaction effect was noted.

Fear of Publicly Observable Anxiety Reactions. A $2 \times 8$ repeated measures ANOVA of the Fear of Publicly Observable Anxiety Reactions subscale also indicated a significant main effect for session, $F(7,154)=2.23, p=.04, n 2_{p}=.09$ : On average, scores at baseline were significantly higher than at Session 1, though scores appeared to return to near baseline levels by post and follow-up. Although no significant main effect for group emerged, a 
significant interaction effect was indicated, $F(7,154)=3.06, p=.005, n 2_{p}=.12: A$ significant simple effect for session emerged for the exercise group, $F(7,77)=3.84, p=$ $.001, \mathrm{n} 2 \mathrm{p}=.26$; with exercise group scores significantly declining from baseline to the end of Session 1 and continuing to decline through post and follow-up. In contrast, scores for the control group did not significantly change over time $(p>.10)$.

Fear of Cardiovascular Symptoms. A 2 × 8 repeated measures ANOVA of the Fear of Cardiovascular Symptoms also indicated a significant main effect for session, $F(7,154)=$ $2.23, \mathrm{p}=.04, \mathrm{n} 2_{\mathrm{p}}=.09$, with scores again significantly decreasing from baseline to post Session 1 and remaining lower through post and follow-up. A group by time interaction effect was also found, $F(7,154)=3.46, p=.002, n 2_{p}=.14$. A significant simple effect for assessment session emerged for the exercise group, $F(7,77)=4.83, p<.001, n 2_{p}=.31$, indicating that the decline in scores from baseline to post Session 1 was significant, with scores remaining significantly lower through post and follow-up. No simple effect was noted for the control group ( $p>.10)$.

Fear of Cognitive Dyscontrol. A $2 \times 8$ repeated measures ANOVA of the Fear of Cognitive Dyscontrol subscale indicated a significant main effect for group, $F(1,22)=6.28, p=.02$, $\mathrm{n} 2_{\mathrm{p}}=.22$, with the exercise group scoring significantly lower on average than the control group. No main effect for session was noted. However, the interaction effect was significant, $F(7,154)=4.45, p<.001, n 2_{p}=.17$. Post hoc analyses indicated that exercise group scores significantly declined following the first session and were maintained through follow-up, whereas scores for the control group increased somewhat (though not statistically significant) across the sessions.

\section{DISCUSSION}

Previous research has indicated that repeated exposure to physiological cues in the context of aerobic exercise is associated with reductions in fears of anxiety-related sensations (Broman-Fulks et al., 2004). However, the initial study utilized an active comparison condition, and participants assigned to the low-intensity exercise comparison condition also reported significant decreases in anxiety sensitivity. Thus, regression to the mean could not be ruled out as a potential explanation for their findings. Furthermore, previous research has not directly examined the effects of exercise on the major subcomponents of anxiety sensitivity. In an attempt to build on previous research, the present study compared the effects of aerobic exercise on full- and subscale anxiety sensitivity scores with a no-exercise control condition. The results were consistent with previous research in indicating that exercise-induced exposure to physiological arousal was associated with significant declines in self-reported fears of anxiety sensations. In contrast, individuals assigned to a no-exercise control condition did not report significant changes in full-scale ASI-R scores. Furthermore, whereas aerobic exercise was associated with significant reductions in each of the four ASI-R subscales, suggesting decreased fears of respiratory and cardiovascular symptoms, publicly observable anxiety symptoms, and cognitive dyscontrol, only respiratory symptom fears declined significantly among the control group. Thus, statistical regression does not appear to account for the effects of exercise on anxiety sensitivity scores.

An analysis of the change in anxiety sensitivity scores across the exercise sessions appears to indicate that the decline in scores occurs relatively quickly following the initiation of an exercise program. Specifically, the present study indicated that a single 20-minute bout of aerobic exercise can lead to significant reductions in anxiety sensitivity scores, as well as 
more specific fears of cardiovascular and respiratory symptoms, socially observable anxiety symptoms, and cognitive dyscontrol. These findings are consistent with previous research, which suggested that individuals report less fear of anxiety-related physical sensations following a single exercise session (Broman-Fulks et al., 2004). Thus, the apparent fast acting effects of aerobic exercise may make it a particularly attractive form of intervention for individuals with high anxiety sensitivity who desire rapid reductions in anxiety-related fears and associated phenomena.

Noteworthy are the potential public health implications of this research. Specifically, anxiety disorders are some of the most prevalent mental disorders in the United States, affecting as many as 30\% of the American public (Kessler, Berglund, Demler, Jin, \& Walters, 2005) and costing billions of dollars each year for treatment alone (Greenberg et al., 1999). Elevated anxiety sensitivity has been shown to be associated with increased risk for panic and other anxiety disorders (e.g., Taylor, Koch, \& McNally, 1992), as well as some mood (e.g., Cox, Enns, Freeman, \& Walker, 2001) and substance use disorders (e.g., Stewart, Peterson, \& Pihl, 1995). Thus, interventions that lower anxiety sensitivity have the potential to serve as primary, secondary, or tertiary prevention methods, thereby reducing the risk and incidence of psychopathology. Indeed, recent research appears to indicate that anxiety sensitive individuals who complete anxiety sensitivity prevention programs are less likely to subsequently meet criteria for a variety of Axis I disorders (Schmidt et al., 2007). However, additional prospective research will be necessary to determine the extent to which aerobic exercise-induced reductions in anxiety sensitivity impact the development or course of psychological disorders. If, like other anxiety sensitivity prevention programs, participation in regular physical exercise can be shown to reduce risk of psychological dysfunction, physical exercise may be a particularly attractive form of intervention to clinicians and patients for several reasons. First, exercise is an easily accessible form of intervention for most physically healthy individuals, regardless of geographic location, intellectual capacity, or financial means. Second, exercise participation requires minimal or no clinician contact, thereby saving clinicians and patrons valuable resources (e.g., money, time) that may otherwise be expended on traditional forms of treatment (e.g., psychotherapy or pharmacotherapy). Finally, exercise appears to be a particularly efficient form of intervention, with initial evidence suggesting that a single bout of exercise can significantly reduce anxiety sensitivity.

Although aerobic exercise appears to reliably reduce self-report anxiety sensitivity, additional research will be necessary to clarify several important issues. For example, because anxiety sensitivity scores appear to decline following a single bout of exercise, it will be beneficial for future research to compare the relative efficacy of a single bout of exercise with more frequent exercise participation. Furthermore, the relative duration of the anxiolytic effect of exercise following cessation of exercise remains unclear. To date, research appears to indicate that improvements in anxiety sensitivity are maintained for at least 1 week following termination of a 2-week exercise protocol (Broman-Fulks et al., 2004). Relatedly, a recent investigation of a brief CBT prevention program for anxiety sensitivity indicated that completion of a one-session CBT protocol was associated with reduced risk for Axis I disorders at a 2-year follow-up (Schmidt et al., 2007). However, the robustness of anxiety sensitivity reductions following the discontinuation of single or multiple bouts of exercise remains to be investigated. It is also plausible that alternative forms of exercise (e.g., weight training, yoga, sports) may confer similar benefits to those of aerobic exercise, though this remains to be empirically tested. Finally, a direct comparison of aerobic exercise with a well-established form of treatment for anxiety sensitivity-related 
problems (e.g., CBT) would help to further evaluate the potential clinical utility of exercise as an intervention or component of efficacious treatment for anxiety sensitivity and related psychopathology.

Although the present research was designed to address some of the methodological concerns identified in the initial investigation into the effects of aerobic exercise on anxiety sensitivity, several limitations of the current research influence the strength with which conclusions can be drawn. For example, the present study utilized a relatively small sample of non-clinical participants with heightened anxiety sensitivity. Thus, the generalizability of these findings to individuals with various psychological conditions known to be associated with pathological levels of anxiety sensitivity (e.g., panic disorder, posttraumatic stress disorder, major depression) remains unclear. However, it should be noted that the present study did not intentionally exclude individuals with concurrent psychopathology, and indeed, $25 \%$ of participants reported a history of panic attacks. Regardless, replication of exercise-induced reductions in anxiety sensitivity among a clinical sample would greatly improve the credence of these findings and provide additional impetus for considering aerobic exercise as a potential treatment for high anxiety sensitivity and associated conditions. Furthermore, long-term follow-up with clinical participants would enable researchers to clarify whether exercise-induced reductions in anxiety sensitivity are associated with corresponding reductions in risk for panic and other Axis I disorders, which, at present, remains unclear.

A second limitation of the present study that researchers may want to address in future studies was the lack of attention control. On average, exercise participants spent 25_30 minutes in the laboratory for each exercise session, whereas participants in the no-exercise control condition only spent a few minutes completing the ASI-R. Thus, it is possible that non-specific effects resulting from the differential time requirements for the groups could have influenced these findings. In an attempt to minimize the potential influence of researcher attention, the present study limited contact between the researcher and exercise participants. Specifically, researchers monitored exercise participants from a separate room, only entering the exercise room to record heart rates and adjust treadmill speeds if necessary. Future researchers may wish to consider standardizing time spent in the laboratory, or implementing other non-exercise active control conditions, to reduce concerns over alternative influences on anxiety sensitivity score reductions.

A third potentially influential variable not controlled for in the present study is possible changes in participants' exercise habits that may have occurred outside of the experimental context. Although the study was relatively brief and the inclusion criteria mandated that participants could not be currently involved in an exercise program, it is possible that some participants could have elected to begin an external exercise regimen during the 3 weeks they were being followed by the current research. To address this potential limitation, future research would benefit by systematically evaluating and controlling for changes in exercise habits that occur during exercise treatment protocols.

Finally, it should be noted that this study represents the first examination of the effects of exercise on the dimensions of anxiety sensitivity. Because the analyses of the ASI-R subscales were exploratory in nature and the sample was relatively limited in size, no adjustment of alpha levels was made in the present research, thereby inflating the risk of a Type I error among these analyses. Thus, these findings should be interpreted with caution until future research is able to more thoroughly investigate the effects of physical exercise on the subcomponents of anxiety sensitivity. 
In sum, aerobic exercise participation appears to yield significant reductions in self-report anxiety sensitivity. Although these results are promising and suggest additional consideration of aerobic exercise as a stand-alone intervention or component of efficacious treatment for individuals with high anxiety sensitivity, considerable research remains to be done. Future research addressing some of the limitations of previous studies and analyzing various manipulations of the exercise methodology and research design will further enhance our confidence in the legitimacy of physical exercise as a viable treatment option for high anxiety sensitivity and associated forms of psychopathology.

\section{ACKNOWLEDGEMENTS}

The authors are grateful to Bunmi Olatunji, Carmen Bondy, Angelica Marsic, Jessica Harris, Jennie Bollinger, Lindsey Helden, and David Naff for their assistance with this study.

\section{REFERENCES}

Abadie, B. R. (1988). Relating trait anxiety to perceived physical fitness. Perceptual and Motor Skills, 67, 539_543.

American College of Sports Medicine (2000). ACSMs guidelines for exercise testing and prescription (6th edn). Baltimore: Lippincott Williams and Wilkins.

Beck, J. G., \& Wolf, M. S. (2001). Response to repeated CO2 in individuals with elevated anxiety sensitivity: Replication with $20 \%$ CO2. Journal of Behavior Therapy and Experimental Psychiatry, 32, 1-16.

Broman-Fulks, J. J., Berman, M. E., Rabian, B., \& Webster, M. J. (2004). Effects of aerobic exercise on anxiety sensitivity. Behaviour Research and Therapy, 42, 125-136.

Cox, B. J., Enns, M. W., Freeman, P., \& Walker, J. R. (2001). Anxiety sensitivity and major depression: Examination of affective state dependence. Behaviour Research and Therapy, 39, 1349-1356.

Deacon, B. J., \& Abramowitz, J. S. (2006). A pilot study of two-day cognitive-behavioral therapy for panic disorder. Behaviour Research and Therapy, 44, 807-817.

Deacon, B. J., Abramowitz, J. S., Woods, C. M., \& Tolin, D. F. (2003). The Anxiety Sensitivity Index-Revised: Psychometric properties and factor structure in two nonclinical samples. Behaviour Research and Therapy, 41, 1427-1449.

Ehlers, A. (1995). A one-year prospective study of panic attacks: Clinical course and factors associated with maintenance. Journal of Abnormal Psychology, 104, 164-172.

Fremont, J., \& Craighead, L. W. (1987). Aerobic exercise and cognitive therapy in the treatment of dysphoric moods. Cognitive Therapy and Research, 11, 241-251. 
Greenberg, P. E., Sisitsky, T., Kessler, R. C., Finkelstein, S. N., Berndt, E. R., Davidson, J. R., et al. (1999). The economic burden of anxiety disorders in the 1990s. Journal of Clinical Psychiatry, 64, 1465-1475.

Kessler, R. C., Berglund, P., Demler, O., Jin, R., \& Walters, E. E. (2005). Lifetime prevalence and age-of-onset distributions of DSM-IV disorders in the National Comorbidity Survey Replication. Archives of General Psychiatry, 62, 593-602.

McEntee, D. J., \& Haglin, R. P. (1999). Cognitive group therapy and aerobic exercise in the treatment of anxiety. Journal of College Student Psychotherapy, 13, 37-55.

McNally, R. J., \& Lorenz, M. (1987). Anxiety sensitivity in agoraphobics. Journal of Behavior Therapy and Experimental Psychiatry, 18, 3-11.

Salmon, P. (2001). Effects of physical exercise on anxiety, depression, and sensitivity to stress: A unifying theory. Clinical Psychology Review, 21, 33-61.

Schmidt, N. B., Eggleston, A. M., Woolaway-Bickel, K., Fitzpatrick, K. K., Vasey, M. W., \& Richey, J. A. (2007). Anxiety Sensitivity Amelioration Training (ASAT): A longitudinal primary prevention program targeting cognitive vulnerability. Journal of Anxiety Disorders, 21, 302-319.

Schmidt, N. B., Lerew, D. R., \& Jackson, R. J. (1997). The role of anxiety sensitivity in the pathogenesis of panic: Prospective evaluation of spontaneous panic attacks during acute stress. Journal of Abnormal Psychology, 106, 355-364.

Schmidt, N. B., Zvolensky, M. J., \& Maner, J. K. (2006). Anxiety sensitivity: Prospective prediction of panic attacks and Axis I pathology. Journal of Psychiatric Research, 40, 691-699.

Shephard, R. J., Cox, M. H., \& Simper, K. (1981). An analysis of "PAR-Q" responses in an office population. Canadian Journal of Public Health, 72, 37-40.

Stewart, S. H., Peterson, J. B., \& Pihl, R. O. (1995). Anxiety sensitivity and self-reported alcohol consumption rates in university women. Journal of Anxiety Disorders, 9, 283_292.

Taylor., S., \& Cox, B. J. (1998). An expanded Anxiety Sensitivity Index: Evidence for a hierarchic structure in a clinical sample. Journal of Anxiety Disorders, 12, 463-483.

Taylor, S., Koch, W. J., \& McNally, R. J. (1992). How does anxiety sensitivity vary across the anxiety disorders? Journal of Anxiety Disorders, 6, 249-259.

Telch, M. J., Lucas, J. A., Schmidt, N. B., Hanna, H. H., Jaimez, T. L., \& Lucas, R. A. (1993). Group cognitive-behavioral treatment of panic disorder. Behavior Research and Therapy, 31, 279-287. 